\title{
Recombinase-mediated cassette exchange (RMCE) and BAC engineering via VCre/VloxP and SCre/SloxP systems
}

\author{
Sachiko Minorikawa ${ }^{1}$ and Manabu Nakayama ${ }^{1,2}$ \\ ${ }^{1}$ Department of Human Genome Research, Kazusa DNA Research Institute, Chiba, Japan, and ${ }^{2}$ Laboratory of \\ Pharmacogenomics, Graduate School of Pharmaceutical Sciences, Chiba University, Chiba, Japan
}

BioTechniques 50:235-246 (April 2011) doi 10.2144/000113649

Keywords: Site-specific recombination; Genome engineering; Cre homologue; VCre/VloxP; SCre/SloxP; Red recombination; mutant analysis; Nanog

Supplementary material for this article is available at www.BioTechniques.com/article/113649.

Site-specific recombination is a powerful biotechnological tool for genome engineering. We previously reported two novel site-specific recombination systems, VCre/VloxP and SCre/SloxP, that do not cross-react with Cre/loxP and Flp/FRT in culture cells and mouse embryonic stem (ES) cells. In this study, a site-specific recombination assay in Escherichia coli was used to examine the activity of mutant VCre (H314L and Y349F) and mutant SCre (H317L and $\mathrm{Y} 352 \mathrm{~F}$ ), in which both mutated residues lie within the active center of Cre recombination. The site-specific recombination activity of both mutants was significantly decreased. Recombinase-mediated cassette exchange (RMCE) using VloxP and the Vlox2272 mutant site was performed in E. coli by introducing a cassette bearing VloxP and Vlox2272 into a recipient plasmid bearing the same sites. RMCE using SloxP and Slox2272 was also performed by SCre recombinase. Moreover, BAC engineering via Red recombination and VCre/VloxP were demonstrated. First, the DNA cassette for modification was introduced into a BAC clone via Red recombination; second, the antibiotics resistance gene flanked by VloxP was removed from the BAC clone by induction of VCre recombinase. Such site-specific recombination systems may effectively be used in combination with other site-specific recombination systems or engineering tools (e.g., Red recombination).

Site-specific recombination that rearranges DNA segments is a powerful biotechnological tool that has been used to develop various methods, such as genome engineering involving conditional knockout, chromosome translocation, introduction of a foreign gene cassette into a specific genome site, and control of gene expression (1). Two popular tools used in genomic engineering are the Cre/loxP and Flp/FRT site-specific recombination systems, which are often used to develop conditional knockout mice. For example, Cre/loxP is used to knock out exon(s) in genes of interest, whereas Flp/ FRT is used to remove neo cassettes of positive selection markers so that normal gene expression remains intact. Cre recombinase, which belongs to the tyrosine recombinase family, originates from P1 phage and recognizes the loxP site, which is composed of an 8-bp sequence flanked on either side by two 13-bp inverted repeats to form a 13-8-13 structure. Cre recombinase can excise a region of DNA surrounded by two loxP sites in the absence of cofactors (2).
Also belonging to the tyrosine recombinase family is Flp recombinase, which originates from yeast plasmid $2 \mu$ and recognizes distinct FRT sites and has the same 13-8-13 base-pair structure as loxP sites. However, the recombination efficiency of the Flp/FRT system is less than that of the Cre/loxP system. To improve recombination efficiency, some researchers have attempted to optimize Flp by introducing mutations that convert it into a thermostable FLP mutant (3) and by altering its codon usage (4). Additionally, there are currently other genomic engineering tools available such as Dre recombinase (5), which recognizes the 32-bp long rox site, and PhiC31 (6) and R4 (7) integrases.

As reported previously (8), we produced two site-specific recombination systems named VCre/VloxP and SCre/SloxP. VCre recombinase and its recognition site, VloxP, originates from plasmid p0908 of Vibrio sp. VCre showed very weak similarity to Cre, sharing $29 \%$ identity to the Cre amino acid sequence. SCre recombinase and its recog- nition site, SloxP, originate from plasmid 1 of Shewanella sp. ANA-3. SCre also showed weak similarity to Cre, sharing $31 \%$ identity to the Cre amino acid sequence and $40 \%$ identity to the VCre amino acid sequence. The VloxP and Slox P recognition sites differ from loxP because VCre and SCre recombinases share low protein similarity with Cre, even though the basic 13-8-13 structures of their recognition sites are identical. Mutant VloxP (e.g., Vlox2272, Vlox43R, and Vlox 43L) and SloxP (e.g., Slox2272, SloxV1R, and SloxV1L), which have the same uses as mutant loxP were also developed (8). VCre/VloxP and SCre/SloxP in combination with Cre/loxP and Flp/FRT systems can serve as powerful tools to genetically modify both alleles of a single gene in mouse ES cells and human iPS cells (8).

In this study, we used amino acid substitution analysis to examine the similarity of VCre and SCre with Cre recombinase. We also examined whether some of the methods developed with Cre/loxP can be 


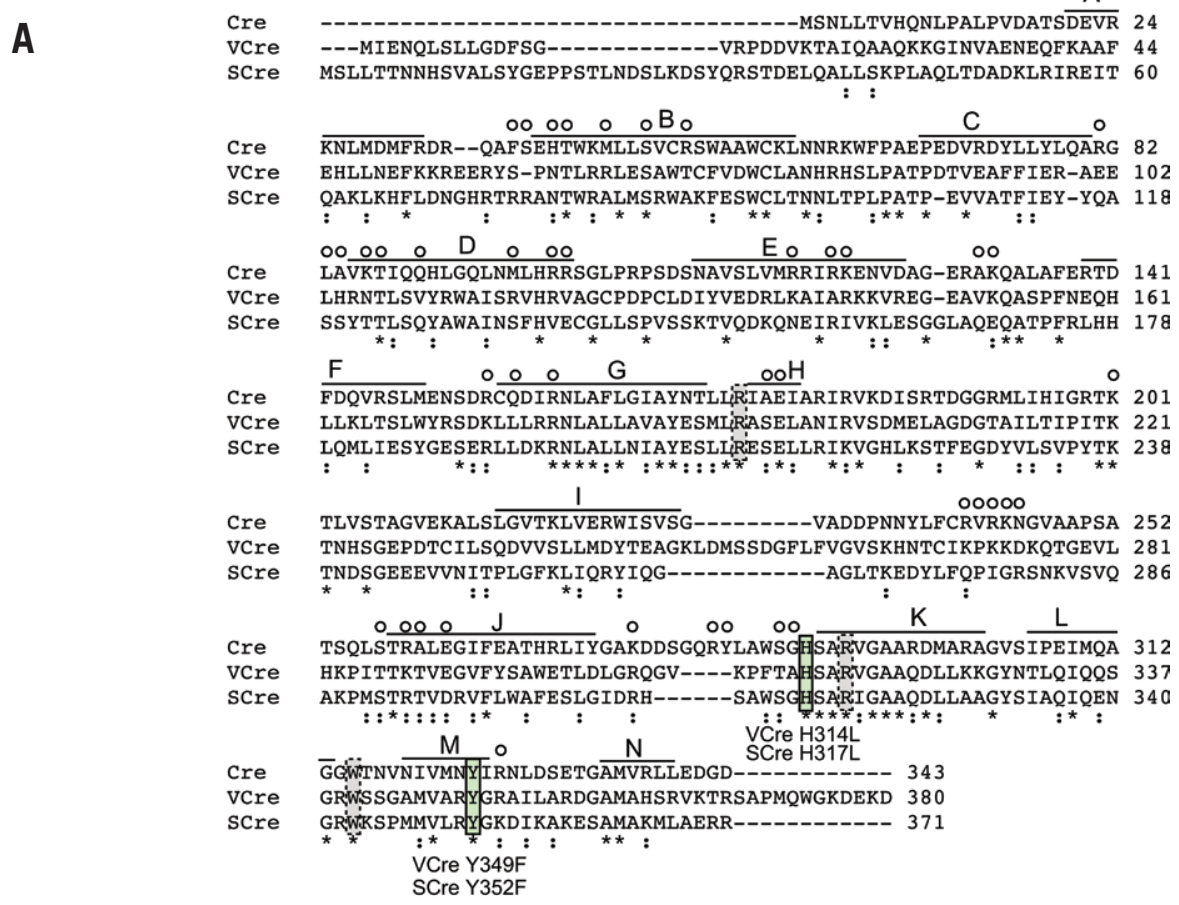

B

\author{
ATAACTTCGTATA GCATACAT TATACGAAGTTAT loxP \\ TCAATTTCTGAGA ACTGTCAT TCTCGGAATTGA VIOxP \\ CTCGTGTCCGATA ACTGTAAT TATCGGACA GAT SlOXP \\ CTCGTGTCCGATA ACTGTAAT TATCGGACACGAG SloxM1 \\ TCAATTTCTGAGA AGTGTCTT TCTCGGAAATTGA Vlox2272 \\ CTCGTGTCCGATA AGTGTATT TATCGGACATGAT Slox 2272
}

Figure 1. Comparison of VCre, SCre, and Cre recombinases and their recognition sites. (A) Alignment of amino acid sequences of VCre, SCre, and Cre recombinases. Alignment was analyzed by ClustalW. The asterisks indicate a perfect match among the three recombinases. Colons (:) indicate similar amino acids. Broken and solid rectangles indicate amino acids at the active center, which was established by the study of Cre recombinase (2). Solid rectangles indicate amino acids that were substituted in this study. The regions of the a helix structure, which was established by the study of Cre recombinase (2), are labeled A-N. The amino acids that contact DNA of the recognition site are indicated with circles above the amino acid sequence. Two amino acid substitution mutants of VCre recombinase, H314L and Y349F, and two amino acid substitution mutants of SCre recombinase, H317L and Y352F, were constructed. (B) Alignment of the recognition sites_VloxP, SloxP, and loxP_of VCre, SCre, and Cre recombinases, respectively. Identical bases among VloxP, SloxP, and loxP are highlighted in yellow. Blue letters indicate mismatched bases within the 13-bp inverted repeat. SloxM1 is a variant recognition site of SloxP. SloxP is composed of a set of 13-bp inverted repeats separated by $8 \mathrm{bp}$. Unlike loxP, the 13-bp inverted repeat is not perfectly matched but contains two base mismatches. SloxM1 was modified to have a perfect 13-bp match, but it does not contain the ATG sequence, which could potentially be used as an initiation Met codon. Red indicates nucleotide substitution in the SloxM1 site and mutant Vlox2272 and Slox2272 sites.

carried out in the newer site-specific recombination systems.

\section{Materials and methods}

Construction of temperaturesensitive plasmid-expressing mutant VCre in $E$. coli

Plasmid pSIM18 was a gift from Donald L. Court (National Cancer Institute-Frederick, Fredrick, MD, USA) (9). Plasmid pSIM18 contains a temperature-sensitive, low-copy plasmid derived from pSC101 and carries an expression system under the control of the lambda pL promoter, the cI857 temperaturesensitive repressor and a hygromycin resistance gene. Following the procedure reported previously (8), we constructed pSIMVCre, pSIMVCreH314L, and pSIMVCreY349F expression plasmids by inserting VCre and mutant VCre genes under the control of the $\mathrm{pL}$ promoter of $\mathrm{pSIM} 18$. The following oligomers were used to construct plasmids pSIMVCreH314L, pSIMVCreY349F, pSIMSCreH317L, and pSIMSCreY352F, and for the PCR amplification of the VCre substitution mutants $\mathrm{H} 314 \mathrm{~L}$ and Y349F: 5'-GAAGCCCTTCACTGCCCTCAGTGCCAGAGTGGGAG-3' and 5'-CTCCCACTCTGGCACTGAGGGCAGTGAAGGGCTTC-3' for VCreH314L; 5'-AGCCATGGTGGCCAGGTTTGGCAGGGCTATCCTGG-3' and 5'-CCAGGATAGCCCTGCCAA ACCTGGCCACCATGGCT-3' for VCreY349F; 5'-GACACTCTGCT TGGTCTGGAC TC TC TGCTAGAATTGGAGCAGC-3' and 5'-GTCCAGACCAAGCAGAGTGTC-3' for $\mathrm{SCreH} 317 \mathrm{~L}$; and $5^{\prime}-\mathrm{CTCCCA}$ TGATGGTC T TGAGAT T TGGAAAGGACATTAAGGCTAA-3' and $5^{\prime}$-ATCTCAAGACCATCATGGGAG-3' for SCreY352F. To construct expression plasmids pSIMVCreH314L, pSIMVCreY349F, pSIMSCreH317L, and pSIMSCreY352F, the resulting PCR products were digested with $A s c \mathrm{I}$ and Not $\mathrm{I}$ and ligated into the $A s c \mathrm{I}$ and $N o t \mathrm{I}$ sites of the PCR product of the pSIM18 backbone (9).

Most of the tester plasmids were reported previously (8). Briefly, to construct tester plasmid pVloxP-EGFP, we ligated the PCR product VloxP-Bsd (blasticidin resistance gene cassette)-VloxP into the NheI and AgeI site of pEGFP-C3 (Clontech). To construct tester plasmidspVloxPBsd-loxPCmand pVloxPBsdVlox $2272 \mathrm{Cm}$, we ligated the PCR product loxP-Cm (chloramphenicol resistance gene cassette)-loxP and Vlox2272-Cm-Vlox2272, respectively, into the $B a m \mathrm{HI}$ and $X h o I$ site of pVloxP-EGFP. The pVloxAmp-loxPCm, pSloxBsd-loxPCm, and pSloxAmp-loxPCm plasmids were replaced with appropriate recognition sites and antibiotic resistance genes from pVloxBsd-loxPCm. 
A

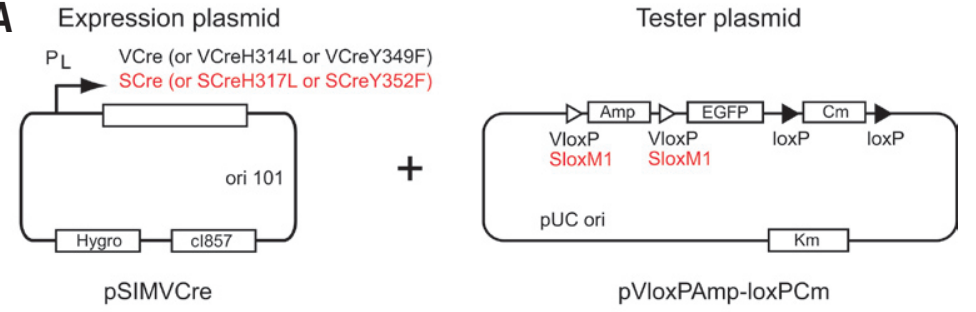

Figure 2. Site-specific recombination of mutant VCre and SCre recombinases in E. coli. (A) Illustration of plasmids expressing mutant $\mathrm{VCre}$ and $\mathrm{SCre}$ recombinases under the control of a temperaturesensitive repressor and tester plasmids for site-specific recombination. (B) Efficiency of site-specific recombination of mutant VCre and SCre. After electroporation of the tester plasmid into $E$. coli DH1OB expressing normal and mutant VCre and mutant SCre, the obtained kanamycin-resistant colonies were replicated on Amp, $\mathrm{Cm}$, or $\mathrm{Km}$ agar plates to test their ability to grow. Empty vector consisted of a backbone plasmid that does not express any recombinase. The efficiency of sitespecific recombination of two mutant VCre proteins was measured. The experiments were performed in triplicate. Error bars, SD.

Site-specific recombination via VCre or SCre expression plasmids in E. coli Site-specific recombination experiments were performed according to the procedure described previously (8). Briefly, E. coli DH10B bearing expression plasmids pSIMVCre, pSIMVCreH314L, and PSIMVCreY349F, which encode VCre or mutant VCre, were grown overnight in Luria-Bertani (LB) medium containing $75 \mathrm{\mu g} / \mathrm{mL}$ hygromycin at $32^{\circ} \mathrm{C}$ with shaking at $170 \mathrm{rpm}$. Fifty microliters of the overnight culture were inoculated into $2 \mathrm{~mL}$ fresh LB medium containing $75 \mu \mathrm{g} / \mathrm{mL}$ hygromycin. The culture was shaken at $32^{\circ} \mathrm{C}$ for $2 \mathrm{~h}$, heated in a $42^{\circ} \mathrm{C}$ water bath for $15 \mathrm{~min}$, and then chilled on ice for $5 \mathrm{~min}$. E. coli cells were transferred into a $1.5-\mathrm{mL}$ Eppendorf tube and centrifuged at $14,000 \times g$ for $30 \mathrm{~s}$. For electroporation, cells were washed three times with cold water and $10 \%$ glycerol, and electroporated with tester plasmid $(0.2 \mu \mathrm{g})$ in $25 \mu \mathrm{L} 10 \%$ glycerol. One milliliter of super optimal broth with catabolite repression (SOC medium) was added to the transformation mixture. Transformants were incubated for $2 \mathrm{~h}$ at $37^{\circ} \mathrm{C}$, and then plated onto an LB agar plate containing $50 \mu \mathrm{g} / \mathrm{mL}$ kanamycin. One hundred colonies selected at random were replicated on LB agar plates containing $100 \mu \mathrm{g} / \mathrm{mL}$ ampicillin, $50 \mu \mathrm{g} / \mathrm{mL}$ kanamycin, or $12.5 \mu \mathrm{g} / \mathrm{mL}$ chloramphenicol. The experiments were performed in triplicate. For SCre experiments, E. coli $\mathrm{DH} 10 \mathrm{~B}$ bearing expression plasmids pSIMSCre, pSIMSCreH317L, and pSIMSCreY352F, which encode SCre or mutant SCre, were used according to the same procedure described in the previous section.

\section{Recombinase-mediated cassette} exchange (RMCE) in $E$. coli As described above, E. coli DH10B cells bearing pSIMVCre were prepared for electroporation after a $42^{\circ} \mathrm{C}$ temperature shift for $15 \mathrm{~min}$. The prepared E. coli cells were electroporated with recipient plasmid, pVloxBsd-Vlox $2272 \mathrm{Cm}$ $(0.2 \mu \mathrm{g})$, and a cassette $(0.1 \mu \mathrm{g})$ bearing an ampicillin resistance gene flanked with VloxP and Vlox2272 in $25 \mu \mathrm{L}$ $10 \%$ glycerol. One milliliter of SOC medium was added to the transformation mixture. Transformants were incubated for $2 \mathrm{~h}$ at $37^{\circ} \mathrm{C}$, and then plated onto an LB agar plate containing $100 \mu \mathrm{g} / \mathrm{mL}$ ampicillin. The plasmids were purified from Amp-resistant colonies, digested with appropriate restriction enzymes, and analyzed by $1 \%$ agarose electrophoresis, and the recombination site was confirmed by DNA sequencing. For RMCE using $S$ Cre recombinase, E. coli $\mathrm{DH} 10 \mathrm{~B}$ cells bearing the pSIMSCre expression plasmid, pSloxBsd-Slox $2272 \mathrm{Cm}$ (as the recipient plasmid), and a cassette $(0.1 \mu \mathrm{g})$ bearing the tetracycline resistance gene flanked with SloxP and Slox 2272 were used. The cassette was produced by PCR amplification using the following primers and pBluescript II SK plasmid as a template: 5' - CCGCTAGCTCAATTTCTGAGAACTGTCATTCTCGGAAATTGATCATGAGACA ATA AC CCTGATAAATGCTTC $-3^{\prime}$ and 5'-CGACCGGTTCAATTTCCGAGAATGACAGTTCTCAGAAATTGATTACCAATGCTTAATCAGTGAGGCACCTAT- $3^{\prime}$ for the ampicillin resistance gene; and 5'-GCTGTACACTCGTGTCCGATAACTGTAATTATCGGACATGATGCTTCATGTTTGACAGCTTATCAT-3' and 5'-CGAGATCTATCATGTCCGATAAATACACTTATCGGACACGAGGCTTCGCGACTAGTTTAAACCTGC-3' for the tetracycline resistance gene in the pBADTcTypeG plasmid (10).



BAC engineering via the VCre/VloxP system following Red recombination in $E$. coli

A BAC clone (RP11-103J24) containing human genomic DNA of the $N A N O G$ gene was purchased from Invitrogen (Carlsbad, CA, USA). The DNA cassette containing the puromycin resistance gene, polyA signal, and $\mathrm{Bsd}$ resistance gene flanked by two VloxP recognition sites for Red recombination was produced by serial PCR amplification using the following primers and pBSPuroBla-lox2272 plasmid as templates: 5'-CCTTAACCTTTTTTCCAGTCCACСТCTTAAATTTTTTCCTCCTCTTCCTCTATACTAACATGGAAGCGGGTAGGCCTTTG-3', 5'-GGCCGCCTCGACCAGCTTCTGATGG-3' for the puromycin resistance gene; 5'-CCATCAGAAGCTGGTCGAGGCGGCCTCAATTTCTGAGAACTGTCATTCTCGGAAATTGAGTTGACAATTAATCATCGGCA-3' and 5'-TTTGGGGACAAGCTGGATCCACACTTCAATTTCCGAGAATGACAGTTCTCAGAAATTGACAGACATGATAAGATACATTG-3' for the $\mathrm{Bsd}$ resistance gene; and 5'-CTTTTCCTTCTGGAGGTCCTATTTCTCTAACATCTTCCAGAAAAGTCTTAAAGCTGCCTTAACCTTTTTTCCAGTCCACC-3' and 5'-ACAAATCACAGGCATAGGTGAAGATTCTTTACAGTCGGATGCTTCAAAGCAAGGCAAGCTTTGGGGACAAGCTGGATCCA-3' for the entire DNA cassette. For Red recombination, pBADTcTypeG (10) was introduced into E. coli $\mathrm{DH} 10 \mathrm{~B}$ containing the $\mathrm{BAC}$ clone. Red recombination was performed following the procedure described previously (10). The 5' outer-side and 3' outerside primers of the introducing cassette for Red recombination were examined by PCR amplification (5'-CTGGACTGAGCT- 
A RMCE (Recombinase-Mediated Cassette Exchange) Vloxp Vlox2272 $\triangle$ Amp
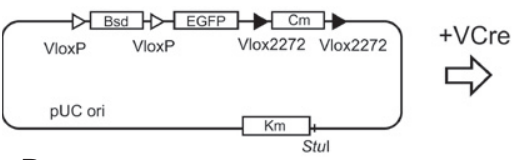

B

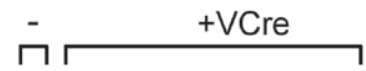

M 1223445567789



C
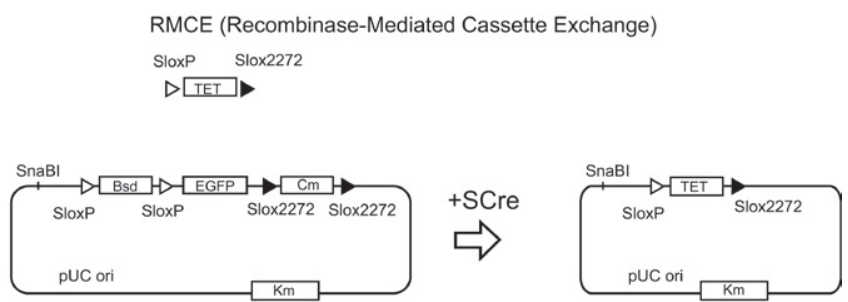

D

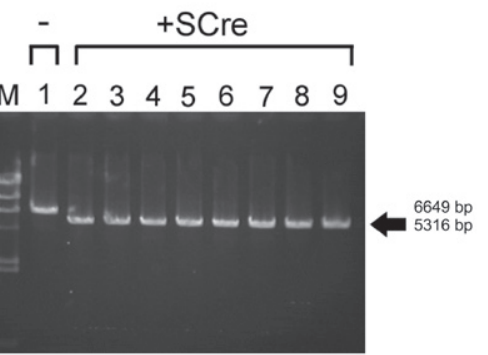

Figure 3. RMCE using VloxP and Vlox2272 or SloxP and Slox2272 in E. coli. (A) Schematic diagram of RMCE using VloxP and Vlox2272. (B) Analysis of RMCE using VloxP and Vlox2272. Recipient plasmid and Amp cassette bearing VloxP and Vlox 2272 were introduced by electroporation into E. coli DH10B expressing VCre. Transformants were grown on Amp agar plates. Plasmids purified from each colony were digested with a single-cutting restriction endonuclease, Stul. Lane 1, control recipient plasmid before the VCre reaction. Lanes 2-9, independently isolated Amp-resistant plasmid after the VCre reaction. $M$, size markers ( $\lambda$ /HindIII; 23, 9.4, 6.6, 4.4, 2.3, and 2.0 kb). Arrow indicates the size of Amp-resistant 4920-bp plasmid after RMCE. (C) Schematic diagram of RMCE using SloxP and Slox2272. (D) Analysis of RMCE using SloxP and Slox2272. Recipient plasmid and the cassette containing tetracycline (TET) resistance gene flanked by SloxP and Slox2272 were introduced into SCre-expressing E. coli DH10B by electroporation. Transformants were grown on TET agar plates. Plasmids purified from each colony were digested with a single-cutting restriction endonuclease, SnaBI. Lane 1, control recipient plasmid before the SCre reaction. Lanes 2-9, independently isolated TET-resistant plasmid after SCre reaction. M, size markers. Arrow indicates the size of TET-resistant 5316-bp plasmid after RMCE.

GGTTGCCTC-3' and 5'-GCGCACCGTGGGCTTGTACTC-3' for 5'-side testing; 5'-CCGCAACCTCCCCTTCTACGA-3' and 5'-GGACATAGTTCAAAGGGCAGG-3' for $3^{\prime}$-side testing), and recombinant $\mathrm{BAC}$ clones were detected.

pSIMVCre was introduced into $E$. coli $\mathrm{DH} 10 \mathrm{~B}$ containing the engineered BAC to remove the Bsd cassette flanked by two VloxP sites. A single colony of E. coli $\mathrm{DH} 10 \mathrm{~B}$ bearing both pSIMVCre and engineered $\mathrm{BAC}$ was grown overnight at $32^{\circ} \mathrm{C}$ in $\mathrm{LB}$ medium containing $75 \mu \mathrm{g} / \mathrm{mL}$ hygromycin and $12.5 \mu \mathrm{g} / \mathrm{mL}$ chloramphenicol. Fifty microliters of the overnight culture were inoculated into $2 \mathrm{~mL}$ of fresh $\mathrm{LB}$ medium containing $75 \mu \mathrm{g} / \mathrm{mL}$ hygromycin and $12.5 \mu \mathrm{g} / \mathrm{mL}$ chloramphenicol, and grown at $32^{\circ} \mathrm{C}$ with shaking for $2 \mathrm{~h}$. The culture tube was heated in a $42^{\circ} \mathrm{C}$ water bath for $15 \mathrm{~min}$, and then chilled on ice for $5 \mathrm{~min}$. E. coli cells were transferred to a 1.5-mL Eppendorf tube and centrifuged at maximum speed for 30 s. Then $E$. coli cells were resuspended in fresh LB medium containing $12.5 \mu \mathrm{g} / \mathrm{mL}$ chloramphenicol. After $8 \mathrm{~h}$ of culturing with shaking, $E$. coli cells were spread with an inoculation loop onto LB agar plates containing $12.5 \mu \mathrm{g} / \mathrm{mL}$ chloramphenicol. Each colony was analyzed by PCR amplification using outer-side PCR primers and Southern blot hybridization as described in the following section.

\section{Southern blot analysis}

Southern blot analysis was performed using the DIG-labeled DNA probe, which was located downstream of the introducing cassette. Southern blot analysis of each $\mathrm{BAC}$ clone was performed using a probe and purified BAC DNAs digested with XhoI or $S p h I$. The probe was generated by PCR amplification from the original BAC clone (RP11-103J24) bearing genomic DNA containing the human NANOG gene using the following oligomers: $3^{\prime}$ probe of $556 \mathrm{bp}, 5^{\prime}$-CACAGAATTCGGCAGTTGAAC-3' and 5'-AAGTGGTAGCGGCTTTATGAG-3'. The isolated recombinant $\mathrm{BAC}$ clones were confirmed by DNA sequencing.

\section{Results and discussion}

The active center residue of VCre and SCre are identical to that of Cre Previously, we described a novel VCre/VloxP and SCre/SloxP system that functions in HEK293 cells, mouse ES cells, and $E$. coli (8). Figure $1 \mathrm{~A}$ demonstrates the similarity between VCre, SCre, and Cre. Even though there was only 30-40\% identity between any two of the amino acid sequences, the active center residues established in the Cre protein (2), namely Cre 173R, 289H, 292R, 315W, and 324Y, were highly conserved. Two small regions of high similarity among the three recom- binases were present at $\alpha$-helix $\mathrm{G}$ and $\mathrm{K}$. Only 5 of 42 amino acid residues were identical among the three recombinases in the 42-amino acid region, which was demonstrated to contact DNA of the recognition site in a three-dimensional structure study of the Cre protein (2). This is also consistent with the fact that the recognition sites of VCre and SCre are partially similar but distinct, with $18 / 34$ and $17 / 34$ identity to loxP, respectively. The identity between VloxP, SloxP, and loxP recognition sites was $12 / 34 \mathrm{bp}$ (Figure 1B). When protein structure homology modeling of VCre or SCre was performed using SWISSMODEL (11), the 3-D structure of these two proteins was found to be highly similar to that of Cre (Supplementary Figure S1).

To determine whether the predicted active center residues of VCre are important for site-specific recombination activity, we introduced amino acid substitutions into VCre to produce mutant recombinase. VCre Y349F is a substitution mutant of a tyrosine residue that, based on Cre studies (2), is responsible for cleavage of DNA at the recognition site to form covalent 3'-phosphotyrosine intermediates. Mutant analysis of Flp and Cre demonstrated that a Leu substitution at His305 of Flp (known as Flp H305L mutant) results in an accumulation of covalent intermediates (12). This effect was also observed for His289 mutants having Gln and Leu 
substitutions at His289 in Cre (13). We constructed $\mathrm{Y} 349 \mathrm{~F}$ and $\mathrm{H} 314 \mathrm{~L}$ mutants of VCre, and $\mathrm{Y} 352 \mathrm{~F}$ and $\mathrm{H} 317 \mathrm{~L}$ mutants of SCre to examine the importance of Tyr349 and His 314 of VCre and Tyr352 and His 317 of SCre.

Mutant VCre and SCre do not recombine at their recognition sites efficiently

Following a procedure described previously (8), we constructed mutant VCre expression plasmid in E. coli. Plasmid pSIM18 (9) was used as a backbone vector. It is of temperature-sensitive origin (ori101), which is compatible with both ColE ori (e.g., pUC) and oriS (e.g., pBeloBAC11 and pBACe3.6). This temperature-sensitive origin is useful for removing the $\mathrm{pSIM}$ plasmid itself after the VCre/VloxP reaction. As shown by Court et al. (9), the $\mathrm{P}_{\mathrm{L}}$ promoter in pSIM is controlled by a temperature-sensitive lambda phage cI857 repressor. We positioned the mutant $V C r e$ gene under the control of this temperature-regulated promoter. Thus, $E$. coli DH10B bearing pSIMVCreH314L or pSIMVCreY349F was maintained at $32^{\circ} \mathrm{C}$, shifted to $42^{\circ} \mathrm{C}$ for $15 \mathrm{~min}$ to induce expression of the VCre mutant, and then maintained continuously at $37^{\circ} \mathrm{C}$ to remove pSIMVCreH314L or pSIMVCreY349F from E. coli $\mathrm{DH} 10 \mathrm{~B}$.

To examine the efficiency of mutant VCre, we electroporated the tester plasmid shown in Figure 2A into competent E. coli $\mathrm{DH} 10 \mathrm{~B}$ cells expressing VCre after temperature-shift at $42^{\circ} \mathrm{C}$ for $15 \mathrm{~min}$. The transformants were grown on $\mathrm{Km}$ plates, and randomly selected colonies were screened on Amp- or Km plates. A tester plasmid, pVloxPAmp-loxPCm, contained an Amp resistance gene flanked by two VloxP sites. Approximately $99 \%$ of the obtained clones in wild-type VCre were Amp sensitive, because the Amp resistance gene was removed via VloxP site-specific recombination. The recombination site in the obtained clones was confirmed by DNA sequencing. Empty vector not expressing any recombinase did not produce clones that were sensitive to Amp. The efficiency of mutant VCre was also examined using the assay described in E. coli. None of the clones obtained using VCreY349F was Amp sensitive, suggesting that the ampicillin resistance gene in the clones was not removed via VloxP. Of the clones obtained using VCreH314L, $1 \%$ was Amp-sensitive. The efficiency of site-specific recombination of mutant VCre was significantly decreased compared with that of wild-type VCre. Interestingly, the number of colonies obtained on $\mathrm{Km}$ plates in the first step using VCreH314L was 1/30 of that of VCreY349F and wild-type VCre.
We expected VCreH314L to lead to an accumulation of the same covalent intermediate as CreH289L and FlpH305 mutants. The formation of such covalent intermediates inhibited plasmid replication, and then reduced the number of colonies after electroporation. These results show that Tyr349 and His314 of VCre play an important role in the active center during site-specific recombination, as they do in Cre recombinase.

Mutant SCre recombinase, SCreH317L, and SCreY352F also lacked recombination activity. As shown in Figure 2B, most of the clones obtained for SCreH317L and SCreY352F, in which no reaction with the SloxP site occurred, were Amp-resistant. About half as many colonies cultured on Km plates were obtained for $\mathrm{SCreH} 317 \mathrm{~L}$ as were obtained for SCreY352F and wild-type SCre. HaloTag fused to the recombinase in each expression system described above was labeled by HaloTag-TMR-ligand and scanned with a Fluorescent Image Analyzer. These quantitative analyses showed that there was no difference in the expression level of any of the recombinases after temperature shift in $E$. coli (data not shown).

Recombinase-mediated cassette exchange via VCre/VloxP and SCre/SloxP systems in $E$. coli

Recombinase-mediated cassette exchange (RMCE) is a useful and highly efficient method of introducing an expression cassette $(14,15)$ or C-terminal protein tag into the genome of ES cells (8). We therefore examined whether VCre/VloxP system is able to facilitate RMCE. We have already described various mutant VloxP sites (8). One mutant site, Vlox2272, exhibits the same feature as lox 2272 of the Cre/loxP system. Vlox 2272 can recombine with itself but cannot react with VloxP. VCre-mediated RMCE was performed in E. coli using VloxP and Vlox2272.E. coli DH10B bearing expression plasmid pSIMVCre was subjected to temperature shift to induce VCre expression, and then was electroporated along with a recipient plasmid (pVloxPBsd-Vlox2272Cm) and a PCR-amplified donor cassette flanked by VloxP and Vlox 2272 sites. Transformants were added to SOC medium, incubated at $37^{\circ} \mathrm{C}$ (restriction temperature) for $2 \mathrm{~h}$ with shaking, and spread on Amp plates. Approximately 10,000 -100,000 Amp-resistant colonies were obtained in a typical experiment. Plasmid DNA was purified from a colony, digested with a single-cutter restriction endonuclease, StuI, and analyzed by agarose gel electrophoresis. As shown in Figure 3B, plasmids from Amp-resistant

\section{BioTechniques}

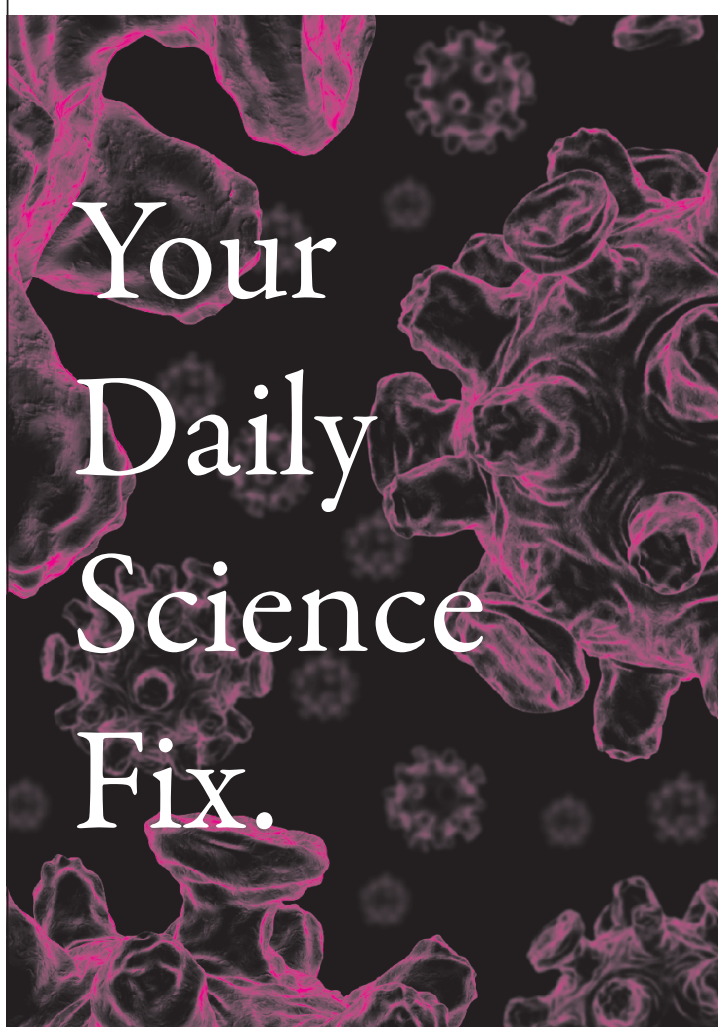

As one of the most comprehensive information sources for biological researchers, the daily newsletter keeps you informed of the most recent developments, products, and industry news in the life sciences. We compile the latest information from the past 24 hours in one convenient source.

Not a subscriber? Sign up for free at: www.BioTechniques.com/newsletters 
A
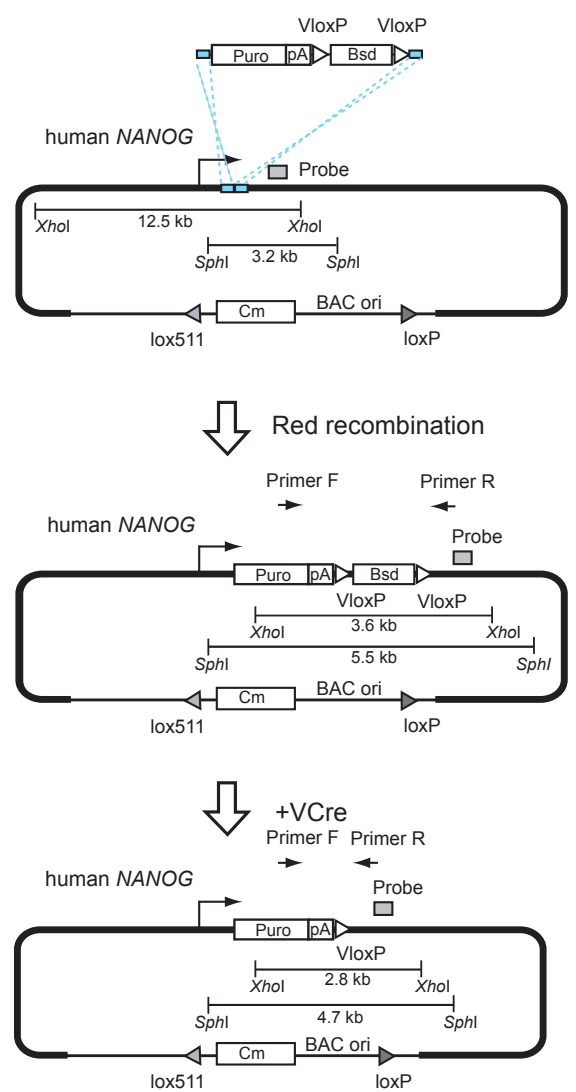

B
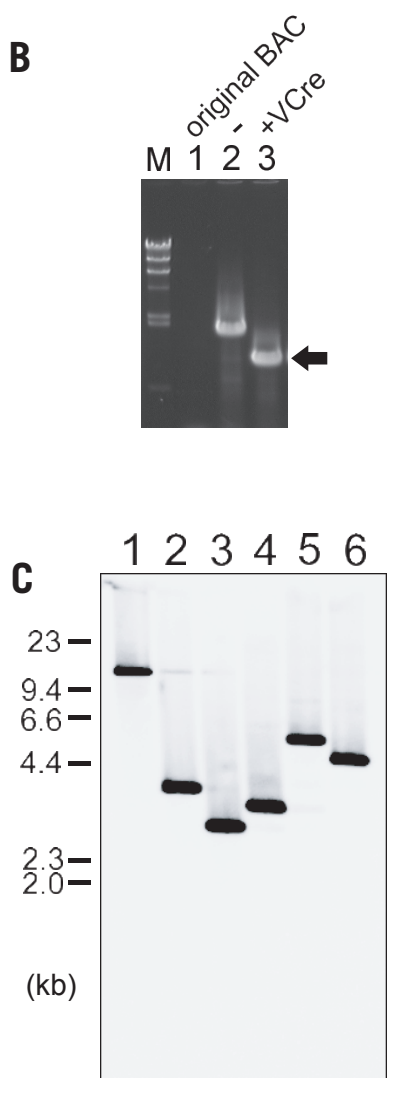

Figure 4. BAC engineering via the VCre/VloxP system following Red recombination. (A) Schematic diagram of BAC engineering via the Red recombination system and site-specific elimination of the antibiotic resistance cassette flanked by VloxP. The antibiotic resistance cassette is needed to engineer BAC by Red recombination; but after Red recombination, the selection marker for Red recombination is no longer required. The Bsd resistance cassette was flanked by two VloxP sites and was removed by VCre. (B) Site-specific elimination of an antibiotic resistance cassette introduced by Red recombination via the VCre/VloxP system. Original (lane 1) and recombinant BACs (lanes 2 and 3) were identified by PCR amplification using primers F and R shown in panel A. The Bsd cassette flanked by two VloxP sequences was removed upon VCre induction (lane 3). Arrow indicates the size of the PCR product, in which the Bsd resistance gene flanked by two VloxP sites was deleted. M, size markers. (C) Southern hybridization analysis of BAC engineering via VCre/VloxP. Purified original BAC (lanes 1 and 4), BACs engineered by Red recombination (lanes 2 and 5), and BACs engineered by VCre (lanes 3 and 6 ) were digested with Xhol (lanes 1-3) or Sphl (lanes 4-6), and then blotted. The probe that localized to the 3' side of the human NANOG gene (see panel A) was used for Southern hybridization analysis.

colonies were shortened by $\sim 1.7 \mathrm{~kb}$ compared with the recipient plasmid. DNA sequencing confirmed that the Amp resistance gene was introduced in place of Bsd-EGFP-Cm of the recipient plasmid, indicating that VCre/VloxP can also be used to perform RMCE. In this experiment, the recipient plasmid has two VloxP and two Vlox2272 sites, because this plasmid was originally designed for another experiment that demonstrated that VloxP does not react with Vlox2272. Due to the presence of two VloxP and two Vlox 2272 sites, four types of replacement products will potentially be produced [i.e., $6816 \mathrm{bp}$ (Bsd-Amp-Cm), 6029 bp (Amp-Cm), 5707 bp (Bsd-Amp), and 4920 bp (Amp) products]. DNA sequencing and agarose gel electrophoresis demonstrated that most of the Amp-resistant colonies were of the shortest type [i.e., $4920 \mathrm{bp}$ (Amp)]. This suggests that even if VloxP or Vlox2272 situated near the egfp gene was used to introduce the Amp cassette during the first stage of RMCE, a second reaction occurred shortly afterward between the VloxP sequences themselves or between the Vlox 2272 sequences themselves. Therefore, most of the plasmids became of the shortest type [i.e., 4920 bp (Amp)]. In this PSIM system, protein induction and plasmid destabilization occurred simultaneously after heat shock. VCre protein was transiently induced and then diluted following segmentation of $E$. coli, because of destabilization of the temperature-sensitive plasmid origin, ori101. This means that although VCre protein only was expressed in E. coli transiently, it was expressed at a level sufficient to support multiple site-specific recombination events over a short period of time. It, however, cannot be excluded that this single RMCE product resulted from the strongly preferable reaction between the two extreme recognition sites.

As shown in Figure 3, C and D, RMCE for SCre recombinase was also performed using SloxP and Slox 2272 sites. The intro- ducing cassette contained a tetracycline resistance gene flanked by SloxP and Slox 2272 . Because of the presence of two SloxP and two Slox2272 sequences in the recipient plasmid, pSloxBsd-Slox2272Cm (6649 $\mathrm{bp})$, four types of replacement products will potentially be produced, namely 7214-bp (Bsd-TET-Cm), 6425-bp (TET-Cm), 6105-bp (Bsd-TET), and 5316-bp (TET) products. DNA sequencing and analysis by agarose electrophoresis demonstrated that all of the TET-resistant colonies were of the shortest type [i.e., 5316 bp (TET)].

\section{BAC engineering via VCre/VloxP}

Next, we examined whether the VCre/VloxP system could be used in BAC engineering. As the BAC clone contained a long DNA insert of $>100 \mathrm{~kb}$, there was no appropriate restriction endonuclease site to modify the BAC clone. Typically, Red recombination is used for engineering of BAC (10). The Red recombination system 
is composed of $\operatorname{Red} \alpha, \beta$, and $\gamma$ originating from lambda phage. Modification occurred via homologous recombination in $E$. coli with a short, 50-60-bp homologous region. Although the Red recombination system is a powerful method for BAC engineering, selection of modified $B A C$ requires the presence of an antibiotic-resistant gene in the modification cassette. After a recombinant BAC has been selected and isolated, the antibiotic resistance gene would no longer be necessary and could potentially disturb gene expression, especially when engineered $\mathrm{BAC}$ is introduced into culture cells or ES cells. A VCre/VloxP system was used to remove the antibiotic resistance gene flanked by VloxP from the BAC. It should be noted that the BAC vector, pBACe3.6, is widely used, but bears lox $\mathrm{P}$ and lox 511. Another popular BAC vector, pBeloBAC11, also has the loxP site. Thus, it is difficult to use the loxP site to remove the antibiotic resistance gene.

Nanog is a popular pluripotent-state marker that is often used as an indicator of the iPS state (16). We engineered BAC containing human genomic DNA with the $N A N O G$ gene to express the puromycin resistance gene as marker under the control of human Nanog promoter/enhancer gene regulation.

As shown in Figure $4 \mathrm{~A}$, the PCR cassette bearing the puromycin resistance gene, polyA, and blasticidin-resistance gene flanked by two VloxP sites contains a 115-bp homologous region at one end and a 84-bp region at the other. This a homologous region-containing cassette was introduced into a BAC clone containing the human $N A N O G$ gene by Red recombination using pBADTcTypeG, as described previously (10). The ATG start codon in the human NANOG gene was replaced with the ATG of the puromycin resistance gene. The cassette was inserted into the human $N A N O G$ gene as is. Engineered BAC was confirmed by PCR using the $5^{\prime}$ primer next to the homologous region and the primer of the puromycin resistance gene. No PCR product was detected using original BAC DNA (Figure 4B, lane 1), but an 1812-bp PCR product was detected using BAC DNA engineered via Red recombination (Figure 4B, lane 2). All engineered regions in the recombinant BACs were confirmed by direct DNA sequencing of PCR products amplified using appropriate PCR primers. Next, pSIMVCre plasmid was transfected into $E$. coli $\mathrm{DH} 10 \mathrm{~B}$ containing the engineered BAC. After induction of VCre recombinase and withdrawing of pSIMVCre plasmid itself by temperature shift, the $E$. coli $\mathrm{DH} 10 \mathrm{~B}$ containing the engineered $\mathrm{BAC}$ was spread onto a plate with an inoculation loop, and some single colonies were analyzed by PCR amplification. The Bsd resistance gene flanked by VloxP was eliminated from all BAC clones, and the PCR products were 787 bp long (e.g., Figure 4B, lane 3). Sitespecific recombination via VloxP was also confirmed by DNA sequencing.

Next, we used Southern blot hybridization to examine $\mathrm{BAC}$ engineering via Red recombination and VCre/VloxP sitespecific recombination. Each type of BAC was purified, digested with $X h o$ I or $S p h I$, and analyzed by agarose electrophoresis. Southern blot hybridization was carried out using the probe shown in Figure 4A. When digested with $X h o \mathrm{I}$, a signal of $12.5 \mathrm{~kb}$ was detected using the original BAC (Figure $4 \mathrm{C}$, lane 1). The BAC engineered via Red recombination produced a signal of $3.6 \mathrm{~kb}$ (Figure $4 \mathrm{C}$, lane 2). The $787 \mathrm{bp}-$ long Bsd resistance gene was eliminated from the $\mathrm{BAC}$ via VCre/VloxP (Figure 4C, lanes 2 and 3). When digested with $S p h I$, a signal of $3.2 \mathrm{~kb}$ was detected using the original BAC (Figure $4 \mathrm{C}$, lane 4). The BAC engineered via Red recombination produced a signal of $5.5 \mathrm{~kb}$ (Figure 4C, lane 5). The signal was 787 bp shorter than that obtained without VCre (Figure 4C, lanes 5 and 6), likely due to $\mathrm{XhoI}$ digestion. No unexpected deletion was detected in the engineered BAC, as there was no difference in the restriction endonuclease digestion product of the original and engineered BAC, except for the modified region of the engineered BAC (data not shown). This suggests that the Bsd resistance gene flanked by two VloxP sites were specifically removed from the BAC without causing any unexpected deletion.

\section{Discussion}

While VCre and SCre are $~ 30 \%$ identical to the amino acid sequences of Cre recombinase, the structure and the amino acids of the active center for site-specific recombination are almost identical. Although these proteins recognize distinct recognition sites, their function is basically identical, indicating that the VCre/VloxP and SCre/ SloxP systems can also be used for RMCE and $\mathrm{BAC}$ engineering.

Many recombination studies have concluded that the site-specific recombination reaction of Cre and Flp recombinases begins with cleavage of the recombining sites at tyrosine residues to form covalent 3 -phophotyrosine linkages to the DNA substrates in the recognition site and release free $5^{\prime}$-hydroxyl groups, indicating that this tyrosine residue is essential for catalysis (2). Substitution to leucine at Tyr349 of VCre and Tyr352 of SCre demonstrated that these tyrosine residues are also essential for sitespecific recombination. Interestingly, the H289L mutant form of Cre, which corre- sponds to H314L of VCre, resulted in the accumulation of covalent intermediates. This mutant phenotype has also been reported for the H305L mutant of Flp (12). In fact, the H314L mutant of VCre exhibits not only a significant decrease in the efficiency of sitespecific recombination, but also a low recovery of colony formation. This phenomenon suggests that a plasmid linked covalently by VCreH314L cannot replicate normally in E. coli $\mathrm{DH} 10 \mathrm{~B}$ lacking the RecA enzyme. This phenomenon is also observed in mutant $\mathrm{H} 317 \mathrm{~L}$ of SCre. Further detailed analysis of the covalent linkages made by VCreH314L and $\mathrm{SCreH} 317 \mathrm{~L}$ are in progress.

Together, mutant analysis of 3-D structure analysis suggest that VCre, SCre, and Cre recombine DNA via the same mechanism but using different recognition sites, even though these three recombinases are 30\% identical to each other. Because three recombinases recombine the DNA via the same mechanism, most methods (e.g., RMCE, BAC engineering, and conditional knockout mice) (1) implementing the Cre/loxP system can thus be carried out in VCre/VloxP and SCre/SloxP systems.

A future direction of research will be the generation and analysis of hybrid proteins composed of VCre, SCre, and Cre. Sitespecific recombination consists of multiple steps, namely recognition of a specific DNA site, formation of covalent 3 '-phophotyrosine linkages to the DNA substrate, ligation of DNA, and-after Holliday junction formation - repeated cutting and ligation of DNA. The large protein region over the entire recombinase protein recognizes the DNA sequence and grips onto it like fingers (2). Producing hybrid proteins consisting of VCre, SCre, and Cre will provide insight into which amino acid sequences in the recombinases define the recognition sites that come into contact with DNA. Ideally, such a hybrid protein derived from VCre, SCre, and Cre would recognize a newly formed recognition site without cross-reacting with VloxP, SloxP, and loxP.

A Flp recombinase mutant that is stable at $37^{\circ} \mathrm{C}$ has been developed to improve recombination efficiency at $37^{\circ} \mathrm{C}$ in order to use cultured cells, ES cells, and mice with maximal recombination efficiency (4). VCre originated from a plasmid derived from Vibrio sp., and SCre originated from a plasmid derived from Shewanella sp., ANA-3. Although we have not yet examined recombination activity at various temperatures, it is highly possible that some amino acid substitutions will improve the recombination efficiency of VCre and SCre at $37^{\circ} \mathrm{C}$. We plan to systematically mutate VCre and SCre to identify variants with improved recombination efficiency. As the most 


\section{Power}

\section{and \\ Performance \\ at your fingertips}

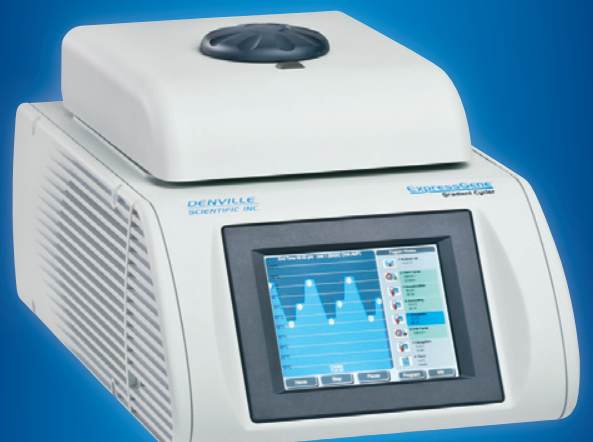

ExpressGene' Gradient Cucler

- Live sensor feedback for superior temperature control

- 96 well gradient thermal cycler

- Large graphical touch screen user interface

- Rapid intuitive "drag and drop" program creation

\section{Only \$3,995}

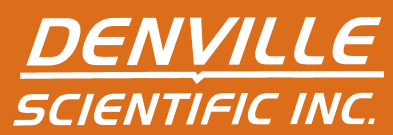

effective application of this technology is in mice, and in animal and human iPS cells, it is important to obtain maximal efficiency of mutant VCre and SCre not only in E. coli, but also in genetically modified murine ES cells and human iPS cells.

As described previously (8), the combined use of VCre/VloxP and SCre/SloxP in addition to Cre/loxP and Flp/FRT is powerful. Now, we have obtained an additional engineering tool for $\mathrm{BAC}$ modification. Since BAC engineering via VCre/VloxP is now available, we can perform complex and sophisticated BAC engineering in combination with Cre/loxP, Flp/FRT, and Red recombination. At present, producing transgenic mice bearing a BAC clone containing a long segment of genomic DNA is the standard means of expressing foreign genes (e.g., cre, egfp, and lacz) under the control of an endogenous promoter/enhancer of a gene of interest. More complex engineering techniques involving BAC clones are available, in which multiple foreign genes are introduced, or in which a single BAC clone is inserted into multiple sites and multiple antibiotic-resistance genes are removed. The VCre/VloxP and SCre/SloxP systems presented here provide an elegant means of performing BAC engineering, the sequential introduction of foreign genes, and a high-throughput approach for introducing multiple modifications into a BAC clone.

\section{Acknowledgments}

We are grateful to Emi Abe for excellent technical assistance. We also thank Osamu Ohara for encouragement. This study was supported by grants from the Kazusa DNA Research Institute, and in part by Grants-inAid for Scientific Research (KAKENHI) from the Japan Society for the Promotion of Science (JSPS).

\section{Competing interests}

The authors declare no competing interests.

\section{References}

1. Branda, C.S. and S.M. Dymecki. 2004. Talking about a revolution: The impact of site-specific recombinases on genetic analyses in mice. Dev. Cell 6:7-28.

2. Guo, F., D.N. Gopaul, and G.D. van Duyne. 1997. Structure of Cre recombinase complexed with DNA in a site-specific recombination synapse. Nature 389:40-46.

3. Rodriguez, C.I., F. Buchholz, J. Galloway, R. Sequerra, J. Kasper, R. Ayala, A.F. Stewart, and S.M. Dymecki. 2000. High-efficiency deleter mice show that FLPe is an alternative to Cre-loxP. Nat. Genet. 25:139-140.
4. Wu, Y., C. Wang, H. Sun, D. LeRoith, and S. Yakar. 2009. High-efficient FLPo deleter mice in C57BL/6J background. PLoS One 4:e8054.

5. Anastassiadis, K., J. Fu, C. Patsch, S. Hu, S. Weidlich, K. Duerschke, F. Buchholz, F. Edenhofer, and A.F. Stewart. 2009. Dre recombinase, like Cre, is a highly efficient site-specific recombinase in E. coli, mammalian cells and mice. Dis Model Mech 2:508-515.

6. Liu, J., T. Skjorringe, T. Gjetting, and T.G. Jensen. 2009. PhiC31 integrase induces a DNA damage response and chromosomal rearrangements in human adult fibroblasts. BMC Biotechnol. 9:31.

7. Liu, Y., B. Thyagarajan, U. Lakshmipathy, H. Xue, P. Lieu, A. Fontes, C.C. MacArthur, K. Scheyhing, et al. 2009. Generation of platform human embryonic stem cell lines that allow efficient targeting at a predetermined genomic location. Stem Cells Dev. 18:1459-1472.

8. Suzuki, E. and M. Nakayama. 2011. VCre/VloxP and SCre/SloxP: new site-specific recombination systems for genome engineering. Nucleic Acids Res. (In press).

9. Chan, W., N. Costantino, R. Li, S.C. Lee, Q. Su, D. Melvin, D.L. Court, and P. Liu. 2007. A recombineering based approach for highthroughput conditional knockout targeting vector construction. Nucleic Acids Res. 35:e64.

10. Nakayama, M. and O. Ohara. 2005. Improvement of recombination efficiency by mutation of red proteins. BioTechniques 38:917924.

11. Kiefer, F., K. Arnold, M. Kunzli, L. Bordoli, and T. Schwede. 2009. The SWISS-MODEL Repository and associated resources. Nucleic Acids Res. 37:D387-D392.

12. Parsons, R.L., P.V. Prasad, R.M. Harshey, and M. Jayaram. 1988. Step-arrest mutants of FLP recombinase: implications for the catalytic mechanism of DNA recombination. Mol. Cell. Biol. 8:3303-3310.

13. Gibb, B., K. Gupta, K. Ghosh, R. Sharp, J. Chen, and G.D. Van Duyne. 2010. Requirements for catalysis in the Cre recombinase active site. Nucleic Acids Res.

14. Schlake, T. and J. Bode. 1994. Use of mutated FLP recognition target (FRT) sites for the exchange of expression cassettes at defined chromosomal loci. Biochemistry 33:1274612751.

15. Bode, J., T. Schlake, M. Iber, D. Schubeler, J. Seibler, E. Snezhkov, and L. Nikolaev. 2000. The transgeneticist's toolbox: novel methods for the targeted modification of eukaryotic genomes. Biol. Chem. 381:801-813.

16. Mitsui, K., Y. Tokuzawa, H. Itoh, K. Segawa, M. Murakami, K. Takahashi, M. Maruyama, M. Maeda, and S. Yamanaka. 2003. The homeoprotein Nanog is required for maintenance of pluripotency in mouse epiblast and ES cells. Cell 113:631-642.

Received 29 November 2010; accepted 14 February 2011.

Address correspondence to Manabu Nakayama, Kazusa DNA Research Institute, 2-6-7 KazusaKamatari, Kisarazu, Chiba 292-0818, Japan. e-mail:nmanabu@kazusa.or.jp

To purchase reprints of this article, contact:

biotechniques@fosterprinting.com 